\title{
Composición, estructura, diversidad e incremento de la vegetación arborea secundaria en trópico seco en Nandarola, Nicaragua
}

\section{Composition, structure, diversity and increased of forest secondary vegetation in dry tropics, Nandarola, Nicaragua}

\author{
Olman Narváez-Espinoza, Benigno González-Rivas, Guillermo Castro-Marín \\ Facultad de Recursos Naturales y del Ambiente, Universidad Nacional Agraria. olmannarvaez@yahoo.com / \\ Benigno.Gonzalez@ci.una.edi.ni / Guillermo.Castro@ci.una.edu.ni
}

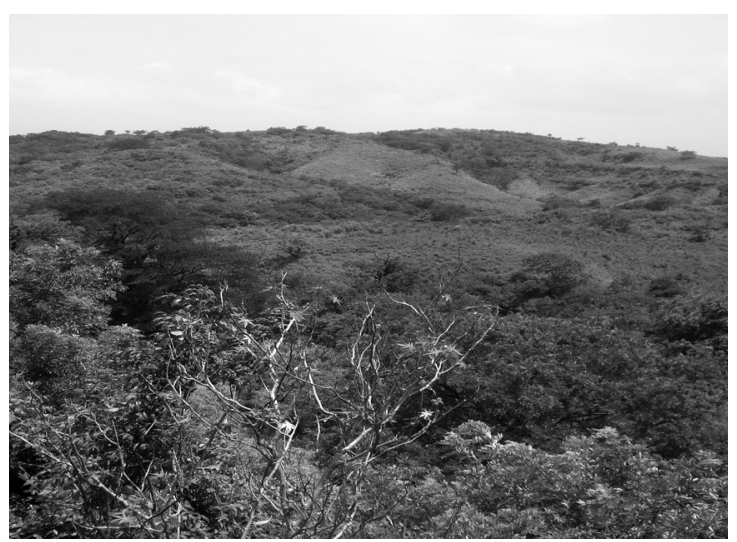

\section{RESUMEN}

Los bosques secos de Nicaragua y sus árboles, presentan una diversidad de bienes y servicios, (medicinales, protección de suelo, fauna silvestre) y belleza escénica, por lo que se requiere de estudios que demuestren la sostenibilidad de los bosques y en específico de las especies que lo habitan. Esta investigación se realizó en la comarca La Chipopa, departamento de Granada, con el objetivo de analizar la composición, estructura, diversidad florística e incremento en la vegetación secundaria del bosque seco tropical para obtener información básica sobre la vegetación arbórea que permita garantizar un manejo ecológicamente sostenible. Para obtener la información se realizaron dos mediciones del diámetro de las especies arbóreas mayores de cinco centímetros en tres parcelas permanentes de 0.25 hectárea entre el periodo 2006 y 2009. Se identificaron 43 especies representadas en 26 familias botánicas, siendo las más representativas Fabaceae, Euphorbeaceae, Hipocrataceae, Mimosaceae, Rubeaceae. La diversidad de especies es un poco mayor en el 2009; confirmando de esta manera que el bosque sigue un proceso lento pero seguro de enriquecimiento florístico. El incremento periódico anual promedio fue de $0.58 \mathrm{~cm}$ año-1. Conforme el tiempo el bosque alcanza una estructura con árboles de todos los tamaños y una mayor composición de especies, siendo muy diversificado y dinámico; sin embargo, se recomienda aplicar tratamientos de raleo y liberación de lianas para favorecer el incremento de las especies, así como, enriquecimiento con especies maderables.

Palabras clave: bosque secundario, sistema policíclico, parámetros demográficos, riqueza y diversidad florística, incremento periódico anual.

Recibido: 10 de febrero 2015

Aceptado: 31 de agosto 2015

\begin{abstract}
The dry forests of Nicaragua present a diversity of goods and services such as timber species as non-timber species (medicinal, floor protection, wild fauna) and scenic beauty. This study was carried out at The Chipopa, community of the department of Granada. The objective was analyzing the floristic composition, structures, floristic diversity and increment, of the secondary vegetation of the tropical dry forest. To obtain basic information on the arboreal vegetation that allows guaranteeing a management ecologically sustainable. There were two mensuration of the diameter of the arboreal species bigger than five centimeters in three permanent plots. The plots were of 0.25 hectare and were established during the period 2006 and 2009. A total of 43 species were identified represented in 26 botanical families, being the most representative Fabaceae, Euphorbeaceae, Hipocrataceae, Mimosaceae, Rubeaceae. The diversity of species is a little bigger in the 2009; confirming in this way that the forest follows a slow but sure process of enrichment floristic. The periodic increment annual average was of $0.58 \mathrm{~cm}$ year- 1 . As the time the forest reaches a structure with trees of all the sizes and a bigger composition of species. It is recommended to apply silvicultural treatments such as lianas liberation, thinning to improve increment of the species.

Keywords: Secondary forest, floristic diversity, periodic increment
\end{abstract} yearly. 
$\mathrm{L}$ os bosques secos tropicales son considerados como los más frágiles, debido a la lenta capacidad de regeneración y a la persistente amenaza de deforestación por causas naturales o antropogénicas (MARENA, 2001).

Como resultado de la deforestación se producen fragmentos de bosques de diferentes tamaños, forma y grado de aislamiento, la disponibilidad y distribución de los recurso forestales son alterados, lo que produce impactos negativos considerables sobre muchas comunidades de plantas y animales (Kattan, 2002).

A pesar de estos factores negativos, los bosques secos en Nicaragua son considerados entre los más diversos y con grandes potenciales de uso por la gama de productos maderables y no maderables.

La mayor parte de los estudios de bosques secundarios han sido realizados en bosques tropicales húmedos (Lamprecht, 1990), lo que hace que existan elementos que limitan el estudio de los bosques tropicales secos como, poco conocimiento de las características florísticas y estructura, así como, de conocimientos básicos silviculturales y ecológicos de las especies.

Conociendo los parámetros de composición, estructura, diversidad e incremento de los bosques tropicales secos, se podrá establecer un mejor uso y manejo de las especies y del bosque en general, que contribuirá al rendimiento sostenido de los recursos naturales, permitiendo tomar decisiones o emitir recomendaciones con las condiciones ecológicas de nuestro propio país, Nicaragua.

\section{MATERIALES Y MÉTODOS}

Área de estudio. Se realizó en la comarca La Chipopa a 12 kilómetros al suroeste de la ciudad de Nandaime en el departamento de Granada, en una finca de 3.8 hectáreas ubicada entre las coordenadas 11 42 '20" y $11^{\circ} 42^{\prime \prime} 31^{\prime}$ de latitud norte y $86^{\circ} 05^{\prime} 01^{\prime \prime}$ y $86^{\circ} 05^{\prime} 10^{\prime \prime}$ de longitud oeste, con elevación media de $151 \mathrm{msnm}$. La vegetación se encuentra en un estado sucesional de 19 años.

La época lluviosa es de mayo a octubre, caracterizándose por una buena distribución en todo el año (Ponce y Montalván, 2005). Según el sistema de clasificación de zonas de vida de Holdridge, pertenece al bosque seco deciduo tropical, con transición a subtropical.

Diseño metodológico. Se realizó en tres parcelas de muestreo permanente de bosque secundario, establecidas en el 2006, por el proyecto Manejo diversificado del bosque secundario en fincas de pequeños productores en Nandarola, Nandaime.

Recolección de datos. Se realizó en el 2009 y una vez colectados los datos se procedió al análisis y comparaciones con la primera medición realizada en el 2006. La recopilación de datos fue a través de un censo de los árboles y arbusto mayor o igual a cinco centímetros de diámetro normal, localizados en parcelas de $50 \mathrm{~m}$ x $50 \mathrm{~m}$.

\section{Variables evaluadas}

Área basal. Es el área de la sección transversal de un árbol medido a 1.30 metros sobre el nivel del suelo y se expresas en metros cuadrados (Prodan et al., 1997). La razón matemática responde a: $\mathrm{Ab}=\pi / 4 *(\mathrm{DN}) 2$

Donde:

$\mathrm{Ab}$ : Área basal expresado en $\mathrm{m}^{2}$

DN: Diámetro normal medido en centímetros

Composición florística. Se evaluó mediante la riqueza de especies (número de especie y familias). La caracterización de la composición del bosque se realizó por medio de lista de la vegetación según el índice de valor de importancia (IVI) de las especies, con base a la población viva al final del estudio. Para este estudio el análisis se realizó en la misma comunidad pero en tiempos diferentes.

Este índice resulta de la suma de los valores relativos de la abundancia, frecuencia y dominancia (Lamprecht, 1990).

IVI $=$ abundancia $\%+$ frecuencia $\%+$ dominancia $\%$

Diversidad de especies. Se evaluó mediante el índice de Shannon-Wiener y el Índice de Simpson

El índice de Shannon-Wiener se determina mediante la siguiente fórmula

$$
\underset{i=1}{\mathrm{H}^{\prime}=-\sum[(\mathrm{n} i / \mathrm{n}) \ln (\mathrm{n} i / \mathrm{n})]}
$$

Donde:

H'= Índice de Shannon-Wiener

$\mathrm{ni}=$ Número de individuos que pertenece a la i- ésima especie en la muestra

$\mathrm{n}=$ Número total de individuos en la muestra

La comparación de la diversidad de especies entre la población del 2006 y 2009 para este índice, se realizó a través de la prueba de hipótesis sobre la similitud o diferencia en la diversidad-abundancia de t Hutchinson, para los índices de Shannon-Wiener, todo esto se realizó mediante el Software Past, estadístico para biólogos (Pérez, 2004).

El índice de Simpson mide la diversidad, basado en las especies más dominantes, toma en cuenta la representatividad de las especies con mayor valor (Pérez, 2004).

$\mathrm{S}$

$$
\begin{aligned}
& \lambda=\sum \text { ni }(\text { ni-1) } / \mathrm{n}(\mathrm{n}-1) \\
& i=1
\end{aligned}
$$


Donde:

$\lambda=$ Índice de Simpson

$\mathrm{ni}=$ Número de individuos que pertenece a la i- ésima especie en la muestra

$\mathrm{n}=$ Número total de individuos en la muestra

Incremento periódico anual (IPA). Para este cálculo se utilizó la fórmula:

$\mathrm{IPA}=[(\mathrm{df}-\mathrm{di}) / \mathrm{t}] / \mathrm{N}^{\mathrm{o}}$

Donde:

IPA = incremento periódico anual del diámetro (cm año-1).

di = diámetro al inicio del periodo

df $=$ diámetro al final del periodo

$\mathrm{t}=$ tiempo entre ambos mediciones (tres año).

$\mathrm{N}^{\mathrm{o}}=$ número de árboles medidos

Análisis estadístico. Se realizó la prueba de t de student, para datos pareados, entre las diferentes especies identificadas en el estudio. Implica datos del 2006 y 2009 dentro de las mismas parcelas de muestreo permanente, con el objetivo de determinar si existen diferencia significativa entre un periodo y otro a nivel de especies, luego de una prueba de normalidad de Shapiro-Wilks y realizando la estandarización de la base de datos con el Logaritmo de base 10. Los datos fueron analizados con el Software estadístico InfoStat.

\section{RESULTADOS Y DISCUSIÓN}

Riqueza y diversidad florística. En la primera medición del 2006, fueron identificadas 40 especies y en 2009, 43 especies, lo que indica tres nuevas especies en un periodo de tres años, Spondias $m o m b i n$, Hippocratea rosea, Salacia macrantha A. C. Sm, esto permite deducir que el bosque seco de Nandarola, está en proceso de enriquecimiento florístico a través del

tiempo, aunque el análisis

estadístico muestre ser no significativo al 5\%.

Estos resultados concuerdan con lo que menciona

Finegan (1992) "que en un bosque secundario joven a medida que se vuelve más maduro, alcanza una estructura con árboles de todo los tamaños y una mayor composición de especies".

Las especies más representativas por poseer el mayor número de árboles corresponden a: Casearia corymbosa,
Gliricidia sepium, Myrospermum frutescens, Lonchocarpus minimiflorus y Stemmadenia obovata, éstas representan para el 2006 el $74.1 \%$ del total de los árboles identificados, siendo estas especies las más comunes para el 2009, solo que esta vez, representan el $71.8 \%$ del total de los individuos.

En la segunda evaluación se encontró una nueva familia botánica (Anacardiaceae), aumentando de 25 a 26 en el 2009. La Fabaceae registró el mayor número de especies (cinco), Euphorbeaceae con cuatro e Hipocrataceae, Mimosaceae y Rubeaceae con tres especies cada una.

Índice de valor de importancia (IVI). En el 2006 las especies ecológicamente más importantes son Gliricidia sepium y Myrospermum frutescens, con 17.3 y 10.4, pero no son las más abundantes quedando determinado su valor de importancia por la dominancia relativa que poseen estas especies.

Las especies G. sepium y $M$. frutescens tienen los mayores valores en aérea basal con tres y $1.2 \mathrm{~m}^{2} \mathrm{ha}^{-1}$, dominando el sitio aunque éstas no son abundantes como Casearia corymbosa.

Tres años después $G$. sepium y $M$. frutescens, continúan siendo las especies ecológicamente más importantes y esta vez son también las más abundantes junto con Lonchocarpus minimiflorus y Stemmadenia obovata, que tienen valores de dominancia relativa altas $(8.3 \%$ y $6.5 \%)$, pero no son más abundantes que C. corymbosa.

Los valores del índice de importancia para las especies G. sepium, M. frutescens, C. corymbosa, L. minimiflorus y $S$. obovata, señalan que están bien representadas en el bosque, por lo que éstas especies, tienen el potencial para recuperar el área basal pérdida, ya sea por la
Cuadro 1. Índice de valor de importancia de las cinco especies más importantes en vegetación secundaria de Nandarola, 2009

\begin{tabular}{|c|c|c|c|c|c|c|c|}
\hline & RUS & Ке 10 & RUS & NeT 70 & Ros & אבר & \\
\hline Gliricidia sepium (Jacq) Kunth ex Walp / Madero Negro & 221.3 & 17.0 & 3.74 & 32.5 & 3 & 3.80 & 18.0 \\
\hline Myrospermum frutescens. Jacq / Chiquirín & 213.3 & 16.3 & 1.45 & 12.6 & 3 & 3.80 & 11.0 \\
\hline Lonchocarpus minimiflorus. Donn / Chaperno & 153.3 & 11.7 & 0.96 & 8.3 & 3 & 3.80 & 8.0 \\
\hline Stemmadenia obovata. Hook. \& Arn / Cachito & 169.3 & 13.0 & 0.75 & 6.5 & 3 & 3.80 & 7.8 \\
\hline Casearia corymbosa. Kunth / Cerillo & 180.0 & 13.8 & 0.66 & 5.7 & 3 & 3.80 & 7.8 \\
\hline Sub total (5 sspecies) & 937.2 & 71.8 & 7.56 & 65.6 & 15 & 19 & 52.6 \\
\hline Otras especies (38 especies) & 369.3 & 28.2 & 3.94 & 34.4 & 64 & 81 & 47.4 \\
\hline Total (43 especies) & 1306.5 & 100 & 11.5 & 100 & 79 & 100 & 100 \\
\hline
\end{tabular}

Abs: Absoluta; Rel: Relativa; IVI: Ídice de valor de importancia. perturbación natural, aprovechamiento o por la aplicación de un tratamiento silvicultural (cuadro 1).

Las especies maderables como Dalbergia retusa (Granadillo), Albizia caribaea (Guanacaste Blanco), Enterolobium cyclocarpum (Guanacaste Negro), Hymenaea courbaril (Guapinol), Cordia alliodora (Laurel) y Bombacopsis quinata (Pochote), muestran índices de valor de importancia muy bajos, oscilando entre $0.5 \%$ y $2.1 \%$. 
Desde el punto de vista económico y ecológico es evidente que estas especies son importantes en el bosque, pero su escasa representatividad en la comunidad las hace vulnerables a perturbaciones naturales y antropogénicas.

Las especies maderables se encuentran en un $90 \%$ en la categoría diamétrica menor (5 a 9.9) por lo tanto, están en competencia con otras especies, lo que sería recomendable realizar una evaluación del grado de competencia a que éstas especies están sujetas mediante una clasificación de para el 2009, el número de árboles por hectárea aumenta en todas las categorías diamétricas, siendo más evidente en la categoría diamétrica mayor $(25 \mathrm{~cm})$, que para la primera medición es de 1 árbol ha-1 y tres años después es de ocho árboles ha-1 como se aprecia en el cuadro 3.

La medida más importante de la organización horizontal es el área basal (Holdridge, 1996), en el bosque de Nandarola muestra un incremento de $2 \mathrm{~m}^{2} \mathrm{ha}^{-1}$ en un periodo de tres años (cuadro 3).

la iluminación y espaciamiento y de Cuadro 3. Distribución del número de individuos y área basal por categorías diamétrica, esta manera determinar el tratamiento mediciones del 2006 y 2009

silvicultural a aplicar para favorecer Clase diamétrica su crecimiento.

\section{Índice de biodiversidad}

Índice Shannon-Wiener. La diversidad de especies es un poco mayor en el 2009; confirmando de esta manera que el bosque de Nandarola,

\begin{tabular}{crrrrrrrr}
\hline Clase diamétrica & \multicolumn{4}{c}{2006} & \multicolumn{4}{c}{2009} \\
\cline { 2 - 10 } & \multicolumn{1}{c}{ Arb ha $^{-1}$} & \multicolumn{1}{c}{$\%$} & $\mathrm{Ab} \mathrm{ha}^{-1}$ & \multicolumn{1}{c}{$\%$} & Arb ha $^{-1}$ & \multicolumn{1}{c}{$\%$} & $\mathrm{Ab} \mathrm{ha}^{-1}$ & \multicolumn{1}{c}{$\%$} \\
\hline $5-9.9$ & 1133.3 & 76.4 & 4.26 & 44.9 & 870.6 & 66.6 & 3.61 & 31.5 \\
$10-14.9$ & 260.0 & 17.5 & 2.90 & 30.6 & 281.3 & 21.5 & 3.31 & 28.8 \\
$15-19.9$ & 70.7 & 4.8 & 1.56 & 16.6 & 100 & 7.6 & 2.36 & 20.0 \\
$20-24.9$ & 17.3 & 1.2 & 0.66 & 6.90 & 46.6 & 3.6 & 1.73 & 15.3 \\
$>25$ & 1.3 & 0.1 & 0.08 & 0.9 & 8.0 & 0.7 & 0.49 & 4.4 \\
\hline Total & 1482.6 & 100 & 9.50 & 100 & 1306.5 & 100 & 11.50 & 100 \\
\hline
\end{tabular}

Árb: Árbol; Ab: Área basal. sigue un proceso lento pero seguro de enriquecimiento florístico. Sin embargo, al realizar la prueba de t Hutchinson para los índices de Shannon-Wiener, resulta ser no significativa en relación al número de especies entre los dos momentos de evaluación ( $p>0.05)$, pero desde el punto de vista ecológico y silvicultural, la diferencia es importante (cuadro 2).

Índice de Simpson. Resultan valores mayores de 0.8 , mostrando una alta expectativa de especies dominantes, esto en los ecosistemas forestales no es apropiado, ya que la composición puede mantenerse relativamente estable por muchas décadas, debido a la dominancia de un pequeño grupo de especies pioneras o heliófitas de larga vida y de poco valor económico (Finegan, 1996; citado por Moraes, 2001).

\begin{tabular}{|c|c|c|}
\hline Índices & 2006 & 2009 \\
\hline $\mathrm{N}^{\circ}$ familias & 25 & 26 \\
\hline $\mathrm{N}^{\mathrm{o}}$ de especies & 40 & 43 \\
\hline $\mathrm{N}^{\mathrm{o}}$ de individuos & 1112 & 979 \\
\hline Shannon-Wiener & 2.48 & 2.55 \\
\hline$t$ de Hutchinson & \multicolumn{2}{|c|}{$\mathrm{t}=1.14$} \\
\hline Para los datos de Shannon & \multicolumn{2}{|c|}{$\mathrm{p}=0.25$} \\
\hline Simpson 1-D & 0.88 & 0.89 \\
\hline
\end{tabular}

Cuadro 2. Prueba de t Hutchinson, entre los índices de ShannonWiener y dominancia de las especies (Simpson)

Estructura horizontal por categoría diamétrica. El mayor número de árboles se encuentran en la categoría diamétrica menor (5 a $9.9 \mathrm{~cm}$ ), 1133 árboles ha-1 para el 2006 y 870 árboles ha-1 en el 2009, contribuyendo de esta manera al $76 \%$ y $66 \%$ respectivamente.

Existen muy pocos árboles con diámetros mayores de 25 centímetros (cuadro 3 ).

A excepción de la categoría diamétrica menor

Las especies Casearia corymbosa y Stemmadenia obovata a pesar de ser las más abundantes no se observan en las categorías dimétricas mayores de 15 centímetros de DAP (díametro a la altura del pecho), debido a que se consideran especies efímeras con forma de vida arbustiva, por lo que no podrán alcanzar mayores diámetros como Gliricidia sepium, que es una heliófita durable y la única que tiene distribución en todas las categorías dimétricas.

Para el año 2009, las especies que alcanzaron la categoría mayor o igual a $25 \mathrm{~cm}$ de diámetro, fueron Gliricidia sepium, Cordia alliodora (Laurel), Pachira quinata (Pochote) y Chlorophora tictoria (mora).

Si la dinámica del crecimiento y la composición se mantienen a este ritmo, estos datos nos permiten predecir, cuando es el momento de aprovechar los árboles de acuerdo al diámetro que se desee; un sistema policíclico será el ideal en el bosque de Nandarola, ya que tenemos distribución de los árboles en todas las categorías diamétricas.

Al estudiar los principales componentes funcionales del bosque como composición y estructura, se determinarán estrategias de conservación y evaluar los impactos de los fragmentos en la vegetación forestal tropical (Matteucci y Colma, 1982).

Incremento periódico anual (IPA). Las especies de mayor incremento diamétrico son Pachira quinata (Pochote) 3.6 cm año ${ }^{-1}$, Bursera simarouba (Jiñocuabo) $1.4 \mathrm{~cm}$ año-1, Cordia alliodora (Laurel), $1.25 \mathrm{~cm}^{\mathrm{año}}{ }^{-1}$, Anona purpurea (Sincoya), 1,2 cm año ${ }^{-1}$ y Karwinskia calderón (Guiliguiste), $1.15 \mathrm{~cm}$ año ${ }^{-1}$, dos de éstas especies son de uso maderable (Pochote y Laurel).

Se debe favorecer el crecimiento de las especies de interés comercial que están surgiendo conforme avanza la sucesión, mediante la aplicación de tratamientos silviculturales o considerarlas en la toma decisiones para el 
plan de manejo del bosque de Nandarola.

Existen diferencias considerables entre el incremento máximo y mínimo de las especies encontradas en el estudio, estas presentan un incremento periódico anual total de $22.7 \mathrm{~cm}$ año ${ }^{-1}$, con un IPA promedio de $0.58 \mathrm{~cm}$ año ${ }^{-1}$.

Las diferencias entre el incremento máximo y mínimo entre la misma especie, es debido principalmente a la vigorosidad del árbol y si este se encuentra oprimido o dominante en el bosque.

El incremento de las especies puede ser variado por el crecimiento alternado de la altura y el grosor del tallo en las diferente categorías diamétricas; así las categorías con diámetros pequeños tienen incremento diamétrico lento, sin embargo, estas categorías pueden estar aumentando en altura, las categorías intermedias parecen destinar recursos para aumentar en diámetros (Klepac, 1983 citado por Finegan y Delgado, 1997).

Finegan y Delgado (1997), mencionan que en la mayoría de las especies, la tasa de crecimiento en altura es mayor que los incrementos en diámetros en las primeras etapas de desarrollo. En cambio, las categorías grandes van disminuyendo su crecimiento gradualmente, llegando a ser muy reducido; confirma también que los valores medios de incremento en diámetro, está fuertemente influenciado por la calidad de la copa, a mayor calidad de la copa mayor incremento.

El incremento periódico anual de las especies maderables es más de dos veces que el de las especies para leña y mayor que el de las especies para forraje, podría ser que las especies maderables, están en pleno crecimiento, además que existen pocos individuos por hectárea, condición que debe tenerse muy en cuenta al momento de realizar un plan de manejo y la aplicación de un determinado tratamiento silvicultural (cuadro 4).

Lo anterior permite aprovechar al máximo el crecimiento, desarrollo y aumento del número de individuos por hectárea de las especies maderables y buscar como el bosque sea autosostenible, ya que contrario al incremento, se cuenta con muy poca área basal de las especies maderables, con relación a la que se tiene de las especies para leña, que es más de 16 veces el área basal.

Cuadro 4. Incremento periódico anual en diámetro por grupos de uso en el bosque de Nandarola

\begin{tabular}{lccc}
\hline Uso & $\begin{array}{c}\text { IPA } \\
\text { cm año }\end{array}$ & $\begin{array}{c}\mathrm{N}^{-} \\
\text {Ind ha }^{-1}\end{array}$ & $\begin{array}{c}\mathrm{Ab} \\
\mathrm{m}^{2} \mathrm{ha}^{-1}\end{array}$ \\
\hline Leña & 4.21 & 1,212 & 9.64 \\
Madera & 9.31 & 24 & 0.56 \\
Forraje & 7.58 & 69.3 & 1.29 \\
\hline
\end{tabular}

En el caso de Nandarola la mayoría de las especies dominantes son efímeras y de uso para leña, éste conocimiento permite tomar la decisión de disminuir la densidad a través de un raleo para favorecer a otras especies evitando la competencia por luz, agua y nutrientes que disminuyen los incrementos, o aprovecharlas como combustible.

\section{CONCLUSIONES}

El estudio de la vegetación en el bosque de Nandarola, indica que existe poca variación en la composición y riqueza florística, identificándose una nueva familia botánica, Anacardiaceae, siendo las familias más representativas Fabaceae, Euphorbeaceae, Hippocrateaceae, Mimosaceae y Rubeaceae, revelando que a medida que el tiempo pasa, alcanza mayor riqueza de especies y diversidad florística.

Las especies más representativas por poseer el mayor número de individuos y ecológicamente más importantes, son Casearia corymbosa, Gliricidia sepium, Myrospermum frutescens, Lonchocarpus minimiflorus y Stemmadenia obovata, obteniéndose tres nuevas especies en el periodo de tres año (Spondias mombin, Hippocratea rosea y Salacia macrantha), demostrando que en un bosque secundario joven a medida que se vuelve más maduro, alcanza una estructura con árboles de todo los tamaños y una mayor composición de especies.

Los valores mayores a 0.8 del índice de Simpson, muestran una alta expectativa de que, dos individuos tomadas al azar sean de la misma especie en las dos mediciones, asegurando que existen especies dominantes.

El incremento en área basal fue altamente significativo, siendo este de $2 \mathrm{~m}^{2} \mathrm{ha}^{-1}$, asegurando la recuperación del bosque en el tiempo, siendo las especies con mayor incremento Pachira quinata con $3.6 \mathrm{~cm}$ año ${ }^{-1}$, Bursera simarouba con $1.39 \mathrm{~cm}^{2}$ ano $^{-1}$, Cordia alliodora con $1.25 \mathrm{~cm}$ año ${ }^{-1}$, y Annona purpurea con $1.17 \mathrm{~cm}$ año ${ }^{-1}$. El incremento periódico anual promedio en diámetro para las especies es de $0.58 \mathrm{~mm}_{\text {año }}{ }^{-1}$.

El manejo estará en dependencia de los productos que ofrece el bosque y éste a su vez, de la composición florística y los incrementos de las especies presentes en el bosque, y posibilita las proyecciones futura para el establecimiento de los ciclo de corto para ciertas especies de acuerdo a su utilidad, el que estaría determinado principalmente por el diámetro. 


\section{REFERENCIAS BIBLIOGRÁFICAS}

Finegan, B. 1992. El potencial de manejo de los bosques húmedos secundarios neotropicales de las tierras bajas. Serie técnica. Informe técnico $\mathrm{N}^{\mathrm{o}} 188$. Colección silvicultura y manejo de bosques naturales. CATIE. Turrialba, CR. 28 p.

Finegan, B; Delgado, D. 1997. Bases ecológicas para el manejo de bosques tropicales: un estudio de caso en Costa Rica. Parte 2. El Chasqui. CATIE 18:16-22.

Holdridge, LA. 1996. Ecología basada en la zona de vida. Instituto Interamericano de Cooperación para la Agricultura. San José, CR. 216 p.

Kattan, G. 2002. Fragmentación: patrones y mecanismos de extinción de especies. In Ecología y conservación de los bosques neotropicales. Libro Universitario regional. Eds. Guariguata, R; Kattan, GH. Cartago, CR. 561 p.

Lamprecht, H. 1990. Silvicultura en los trópicos. Ecosistemas forestales en los bosques tropicales y sus especies arbóreas, posibilidades y métodos para un aprovechamiento sostenido. trad. A. Carrillo. República Federal de Alemania. GTZ. 335 p.

MARENA (Ministerio del Ambiente y los Recursos Naturales, NI). 2001. Informe del estado ambiental en Nicaragua, 2001. Gráfica editora. Manágua, NI. 118 p.

Matteucci, SD; Colma, A. 1982. Metodología para el estudio de la vegetación. Secretaría general de la organización de estados americanos. Washington, US. 168 p.

Moraes Ch. 2001. Almacenamiento de carbono en bosques secundarios en el municipio de San Carlos, Nicaragua. Tesis Mag. Sc. Programa de enseñanza para el desarrollo y la conservación. Escuela de Posgrado. CATIE, Turrialba, CR. 116 p.

Pérez Pérez, AM. 2004. Aspectos conceptuales, análisis numérico, monitoreo y comunicación de datos sobre biodiversidad. MARENA-ARAUCARIA. Centro de malacología/diversidad animal. UCA. Managua. NI. 302 p.

Ponce, L; Montalbán H. 2005. Evaluación del banco de semillas de suelo en tres sitios en diferentes estados sucesionales en un bosque seco secundario en Nandarola, Nandaime, Granada. Tesis Ing. Universidad Nacional Agraria. Managua, NI. 68 p.

Prodan, M; Peters, R; COX, F; Real, P. 1997. Mensura Forestal. Deutsche Gesellschaft für technische Zusammenarbeit (GTZ) $\mathrm{GmbH}$, Instituto Interamericano de Cooperación para la Agricultura (IICA). Serie Investigación y Educación en Desarrollo Sostenible. 586 p. 